\title{
LISA interferometry: recent developments
}

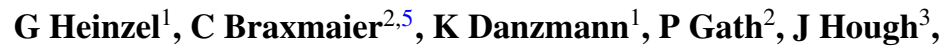 \\ O Jennrich $^{4}$, U Johann ${ }^{2}$, A Rüdiger ${ }^{1}$, M Sallusti $^{4}$ and H Schulte ${ }^{2}$

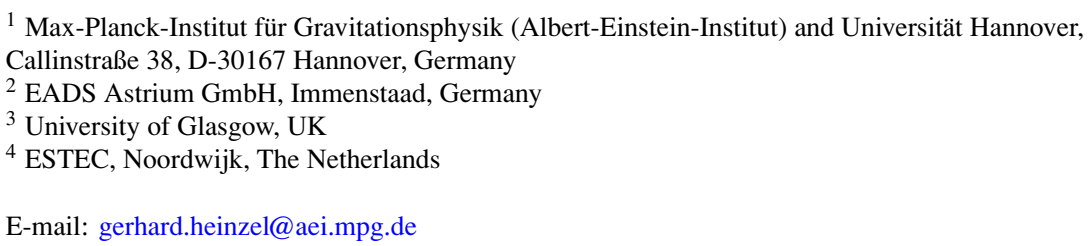

Received 2 October 2005, in final form 15 December 2005

Published 24 March 2006

Online at stacks.iop.org/CQG/23/S119

\begin{abstract}
LISA, the Laser Interferometer Space Antenna, will be launched by NASA and ESA in the 2014 timeframe and will be the first gravitational wave observatory for the many fascinating sources at low frequencies (down to $10^{-5} \mathrm{~Hz}$ ). One of the challenges is to perform precision laser interferometry over a distance of 5 million $\mathrm{km}$. This paper describes recent developments in the interferometer design.
\end{abstract}

PACS numbers: $04.80 . \mathrm{Nn}, 06.30 .-\mathrm{k}$, 07.60.Ly, 07.87.+v, 95.55.Ym

(Some figures in this article are in colour only in the electronic version)

\section{Introduction}

The LISA (Laser Interferometer Space Antenna) mission [1-10] is now firmly entrenched in the planning of both NASA and ESA and is planned to be launched around 2014. LISA consists of three spacecraft forming a triangle with 5 million $\mathrm{km}$ arm length, drag-free control systems with free-floating test masses in each spacecraft and laser interferometry between the spacecraft to measure tiny fluctuations of their distance in the frequency range $10^{-5}-0.1 \mathrm{~Hz}$. Due to orbital mechanics, the spacecraft will move at up to $20 \mathrm{~m} \mathrm{~s}^{-1}$ relative to each other, and the angle will change by about $1.5^{\circ}$ within 1 year. Combined with the fact that only about 100 pW of the emitted laser light arrives at the other end of the arm, this makes the interferometry a challenging enterprise.

Several aspects of the LISA interferometry have previously been studied [11-15], and an industrial study defined a baseline architecture in 2000 [7]. In 2005, a follow-up industrial study is ongoing in order to investigate an updated baseline architecture. This paper reports

5 Present address: University of Applied Sciences, Konstanz, Germany. 


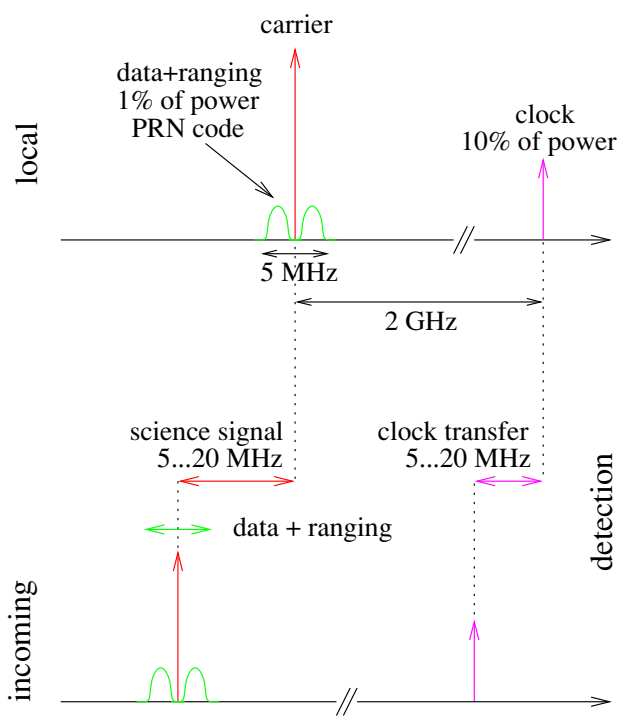

Figure 1. Proposed frequency plan for LISA. Since no heat-producing EOM can be accommodated on the optical bench, the local oscillator light already carries the outgoing modulation. The carrier-carrier beatnote provides the main science measurement. The sideband-sideband beatnote is arranged to be below $20 \mathrm{MHz}$ and provides for the clock comparison. Data transfer and ranging is achieved by spread-spectrum modulation around the carrier. Apart from one master laser which is stabilized to a cavity, all other lasers are phase locked to each other with a frequency offset. By occasional switching of the offset frequencies, all beatnotes can be kept in the range $5-20 \mathrm{MHz}$.

about some new aspects, both from the ongoing study and from other works in the US and Europe.

\section{Frequency plan and laser modulation}

The laser light travelling between the spacecraft has to fulfil several tasks at once: measurement of the carrier phase provides the main science signal (distance fluctuations between spacecraft). In addition, information about the local clocks aboard each spacecraft must be transferred in order for TDI (see below) to work correctly. Assuming that the signal-to-noise ratio is limited by the shot noise on the optical link, it is advantageous to use a frequency as high as possible for this task. While previously a frequency of $200 \mathrm{MHz}$ had been envisaged for this purpose, it turned out that the bandwidth of low-noise photodiodes will be a severe limitation to this approach. The new baseline uses even higher modulation frequencies $(\approx 2 \mathrm{GHz}$ ), but detects the beatnote between the received sideband and the local sideband instead of the sidebandcarrier beat [16]. Thus, a photodiode bandwidth of a few $10 \mathrm{MHz}$ is sufficient. About $10 \%$ of the laser power in these sidebands will provide a sufficient signal-to-noise ratio.

Furthermore, data need to be transferred between the spacecraft and their absolute distance needs to be measured to a few $10 \mathrm{~m}$ accuracy. Both these tasks can be achieved with a weak pseudo-random noise phase modulation of the carrier [17]. With only about $1 \%$ of the light power in these sidebands, both data transfer and ranging can be achieved with the required accuracy. One advantage of this scheme is that orthogonal codes can be used for each link such that no cross-correlations appear, as opposed to ranging schemes with multiple sine tones. Figure 1 shows a diagram of a possible frequency plan. Modulation and demodulation of the 


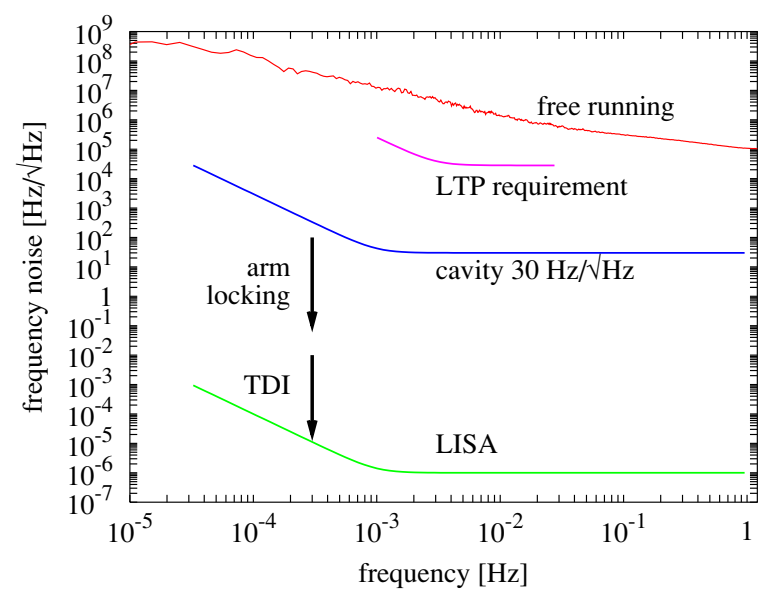

Figure 2. Laser frequency noise levels.

pseudo-random noise is standard practice, apart from the minor difference that here the length of the unambiguous code is longer than the length of one data bit. Typical parameters of the modulation might be a chip rate of $1-2 \mathrm{MHz}$, a data rate (bit rate) of $100-200 \mathrm{kHz}$ and a code length of $100 \mathrm{~km}(300 \mu \mathrm{s})$. The latter will be sufficient for unambiguous ranging, since ranging from the Earth is expected to yield relative positions with $\approx 20 \mathrm{~km}$ accuracy.

The primary measurement consists of measuring the phases of the beatnotes with respect to the spacecraft master clock, after recording them with quadrant photodiodes. Each phasemeter channel could be a relatively simple digital phase-locked loop (PLL) that digitally generates a phase-locked replica of the beatnote. The resulting value of the phase or frequency register is recorded at regular intervals $(3-10 \mathrm{~Hz})$ and is used for the main science output, while the remaining fast phase fluctuations beyond the PLL bandwidth can be processed to recover the pseudo-random modulation.

\section{Laser frequency stabilization}

Due to the enormous arm length differences of $\pm 50000 \mathrm{~km}$ that are caused by the orbital dynamics, laser frequency fluctuations must be suppressed by many orders of magnitude in order not to spoil the measurement. Figure 2 shows (from top to bottom) a typical noise curve of a free-running Nd:YAG laser, the modest frequency stability requirement of the LISA technology package for comparison, the expected noise limit of stabilization to a cavity and the LISA requirement. The present baseline calls for a three-step stabilization scheme. First, one master laser is prestabilized to a reference cavity. The other lasers in the constellation are phase locked with an offset of 5-20 MHz to that master. The next level of stability is achieved by stabilizing the laser frequency to one or more of the long LISA arms. While this requires unusual servo designs, its feasibility has recently been demonstrated [18-23]. The remaining few orders of magnitude cannot be achieved by active stabilization, but only by postprocessing the data with a novel technique called 'time-delay interferometry' (TDI, see [24] and many references therein). For TDI to work, the distances between the spacecraft must be known with an accuracy of a few $10 \mathrm{~m}$ and all phase measurements must be time stamped with an accuracy of a few ns. These requirements are fulfilled by the ranging and clock-comparison scheme described in the previous section. 


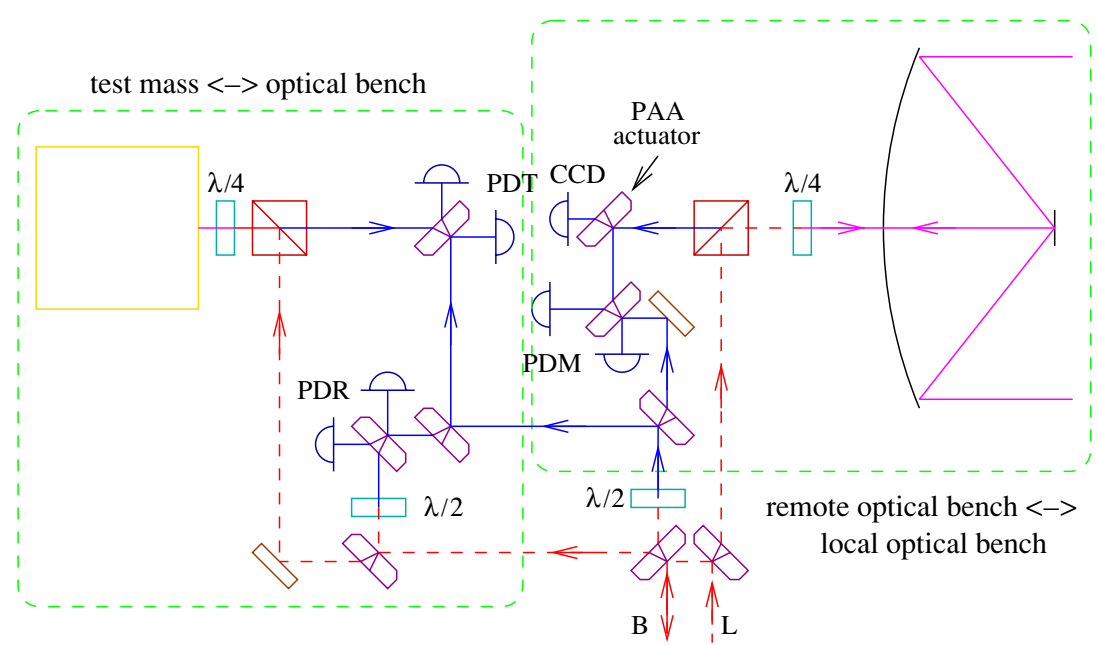

Figure 3. Simplified diagram of one possible layout for the LISA optical bench. The dashed lines indicate separate functions on one single stable optical bench. L represents the local laser that supplies the high-power outgoing beam for this arm. B is connected to the B port of the second optical bench via a fibre. Any light going back to the laser via the $\mathrm{L}$ port is rejected by a Faraday isolator (not shown). The light coming in via the B port is used as local oscillator in the heterodyne interferometers labelled PDM, PDT and PDR that measure the distance to the remote spacecraft, the distance between the local test mass and the local optical bench and a phase reference, respectively. Path length fluctuations of the optical fibre that connects the two benches are cancelled [25]. Many variants are possible, in particular with respect to where the polarizations (shown as dashed (red) and solid (blue) lines in this diagram) are rotated and matched.

\section{Optical bench}

Many layouts for the optical bench have been proposed and discussed in the past. Recent developments propose two important changes: separated interferometry and frequency swapping. In previous layouts, the incoming weak light was first bounced off the test mass before being brought to interference with the local oscillator light. The motivation had been to directly measure the distance between the test masses. In reality, however, it had always been necessary to measure additionally the distance of the test mass to the optical bench ('backside interferometry') and subtract this distance from the primary measurement. The proposed separated interferometry acknowledges this fact and consequently dispenses with reflecting the incoming weak light off the test mass. Instead, it is directly brought to interference on the optical bench, and the distance between test mass and optical bench is separately measured with another interferometer and then subtracted.

This allows the separation of two difficult tasks that were previously closely linked: the optical sensing of the free-floating test mass and the interferometry of the weak light coming in through the telescope with its associated alignment, acquisition and other problems.

Closely related to the separation is the issue of how to deal with the point-ahead angle (PAA). This angle occurs because of the spacecraft motion during the light travel time (33 $\mathrm{s}$ round trip) and amounts to an angle between incoming and outgoing beams of $\approx \pm 6 \mu \mathrm{rad}$ in free space in the vertical plane, varying over 1 year, and a constant $\approx 6 \mu \mathrm{rad}$ in the horizontal plane (referred to the plane of the triangle). Due to the angular magnification of the telescope, the variation is of the order of $1 \mathrm{mrad}$ on the optical bench and needs to be actively compensated. In previous designs, it had been proposed to rotate the test mass for compensation. This led 
to difficulties with the backside interferometer, however. With separated interferometers, the test mass can be left alone and its orientation be optimized for the lowest noise of the drag-free system and its own interferometer.

Another angular actuator is then needed to compensate the point-ahead angle. While the required dynamic range can be achieved with a piezo actuator, the additional path length noise that might be thus introduced is a critical issue and currently under investigation.

The second proposed change to the old baseline layout concerns the local oscillator. Previously, the same laser light that was sent out at high power through the telescope was also used as a local oscillator for the incoming weak light. Any reflection of the outgoing light at telescope components would thus have coherently added to the local oscillator, but with unknown or possibly fluctuating phase. The new idea is to use the second laser on the spacecraft (originally belonging exclusively to the second optical bench for the other arm) as a local oscillator. Since its frequency will be different, any back-reflection from the telescope will no longer produce beatnotes in the measurement band. Figure 3 shows one possible implementation.

\section{Conclusion}

While the principles of LISA interferometry are still the same, recent years have brought a number of improvements that are now the baseline (TDI, arm locking) and some ideas that are still under discussion. It is expected that a consolidated baseline will be settled upon within the near future. At the same time, experiments are beginning in several laboratories worldwide in order to verify different aspects of the interferometry, which is still challenging but seems, after all, feasible.

\section{Acknowledgments}

Many of the results presented here were obtained in the industrial 'Mission formulation' study carried out by Astrium Germany under a contract from ESA/ESTEC.

\section{References}

[1] Jafry Y et al 1994 ESA J. 18 219-28

[2] Danzmann K 1995 17th Texas Symp. on Relativistic Astrophysics and Cosmology vol 759 pp 481-4

[3] Danzmann K 1996 Class. Quantum Grav. 13 A247-50

[4] Danzmann K 1997 Class. Quantum Grav. 14 1399-404

[5] Danzmann K 2000 Fundam. Phys. Space 25 1129-36

[6] Reinhard R 2000 ESA Bull. 103 36-9

[7] Hammesfahr A 2001 Class. Quantum Grav. 18 4045-51

[8] Edwards T et al 2001 Acta Astronaut. 48 549-57

[9] Jennrich O 2002 Nucl. Phys. B 113 282-8

[10] Danzmann K 2003 Class. Quantum Grav. 20 S1-9

[11] Robertson D 1996 Class. Quantum Grav. 13 A271-7

[12] Jennrich O 2002 Class. Quantum Grav. 19 1731-7

[13] McNamara P 2005 Class. Quantum Grav. 22 S243-7

[14] Jennrich O et al 2001 Class. Quantum Grav. 18 4159-64

[15] McNamara P et al 2000 Fundam. Phys. Space 25 1137-41

[16] Folkner W 2003 Unpublished presentation

[17] Parkinson B and Spilker J J Jr (ed) Global Positioning System: Theory and Applications vol 1 (Progress in Astronautics and Aeronautics vol 163) (AIAA)

[18] Sheard B et al 2003 Phys. Lett. A 320 9-21 
[19] Sylvestre J 2004 Phys. Rev. D 70102002

[20] Marin A 2005 Class. Quantum Grav. 22 S235-42

[21] Sheard B 2005 Class. Quantum Grav. 22 S221-6

[22] Thorpe J 2005 Phys. Lett. A 342 199-204

[23] Herz M 2005 Opt. Eng. 44090505 (Preprint gr-qc/0506124)

[24] Tinto M 2005 Phys. Rev. D 71041101

[25] Shaddock D 2005 Personal communication 